\title{
Maintaining Standards in Colorectal Cancer Surgery During the Global Pandemic: A Cohort Study
}

\author{
Julia Merchant ${ }^{1} \cdot$ Ian Lindsey $^{1} \cdot$ David James $^{1} \cdot$ Nick Symons $^{1} \cdot$ Stephen Boyce $^{1} \cdot$ Oliver Jones $^{1}$. \\ Bruce George $^{1} \cdot$ Chris Cunningham ${ }^{1}$
}

Accepted: 20 December 2020/Published online: 9 January 2021

(C) Société Internationale de Chirurgie 2021

\begin{abstract}
Aim Cancer surgery in the COVID-19 pandemic presents many new challenges. For each patient, the risk of contracting COVID-19 during the perioperative period, with the potential for life-threatening sequelae (1), has to be weighed against the risk of delaying treatment. We assessed the response and short-term outcomes from elective colorectal cancer surgery during the pandemic at our institution.

Method We report a prospective cohort study of all elective colorectal surgery cases performed at our Trust during the 11 weeks following the national UK lockdown on 23rd March 2020, compared with the same time period in 2019.

Results Eighty-five colorectal operations were performed during the 2020 (COVID) time period, and 179 performed in the 2019 (non-COVID) time period. A significantly higher proportion of cases during the COVID period were cancer-related $(66 \%$ vs $26 \%, p<0.00001)$. There was no difference in length of hospital stay, complications or readmissions. There were no mortalities in either cohort. Among the cancer patients, there were no differences in TMN staging, R1 resection rate or lymph node yields. No elective patient tested positive for COVID-19 during the perioperative period.

Conclusion At the height of the COVID pandemic, we maintained delivery the of high-quality elective colorectal cancer surgery, with no worsening of short-term outcomes and no compromise in the quality of cancer resections. Ongoing monitoring of this cohort is essential. The risks associated with COVID-19 will continue for some time, necessitating adaptive responses to maintain high-quality cancer services.
\end{abstract}

\section{Introduction}

Following the WHO declaration of a global COVID-19 pandemic on 11th March [1], NHS England recommended suspension of non-urgent elective surgery for 3 months on 17th March, while urging trusts to continue providing emergency surgery and cancer care [2]. However, in many units, cancer surgery and diagnostic services have been

Julia Merchant

juliafmerchant@gmail.com

1 Department of Colorectal Surgery, Oxford University Hospitals NHS Trust, Oxford, UK significantly disrupted. Additionally, nationwide reports suggest a $60-80 \%$ fall in referrals via 2 -week wait pathways $[3,4]$. Predictive modelling by the COVIDsurg collaborative, suggests that over 28 million surgeries worldwide have been cancelled or postponed [5].

Danger to staff and other patients from cross-infection, as well as increased morbidity and mortality by unwittingly performing surgery on COVID-19-positive patients, have naturally been major concerns. Early data from Wuhan suggested a $20 \%$ mortality for patients who after undergoing elective surgery developed COVID-19 [6]. More recently, the COVIDsurg collaborative reported a $26 \%$ 
overall mortality for COVID-positive patients undergoing undifferentiated surgery worldwide [7].

Early in the pandemic, in the absence of precedent, the Royal College of Surgeons and ACPGBI issued cautious clinical guidance $[8,9]$ avoiding anastomoses, reducing laparoscopy and deferring some patients ordinarily offered curative surgery. A recent international study suggests a lack of consensus in several key areas of operative planning and practice, particularly in the assessment of the COVID status of patients preoperatively and the level of risk across a range of procedures [10].

In anticipation of a potential surge in ITU requirements, access to intensive care support for non-COVID-19 patients having major surgery or developing complications might have been severely constrained, complicating the usual care of complex or co-morbid patients.

Lastly, without robust evidence-based guidance, clinicians and managers had to consider the potential for litigation, if decisions were considered ill-advised in retrospect [11].

\section{Methods}

\section{Estate}

At our Trust, we were fortunate to have the capacity to designate a COVID-19 'positive' site for acute patients and a COVID-19 'clean' site for major cancer surgery, while commissioning support from the independent sector for some peripheral cancer surgery. Our breast, endocrine and colorectal services have operated on all suitable patients while providing capacity for other more severely constrained regional Trusts.

\section{Cancer multidisciplinary team (MDT)}

On 16th March, the colorectal MDT agreed some basic principles, updated as national guidelines and outside data were developed, for managing the cancer workload. These consisted of severely curtailing neoadjuvant and adjuvant chemotherapy, substituting long for short-course chemoradiotherapy for rectal cancer and deferring some patients for 3 months: patients with borderline fitness for surgery. Recurrent cancers requiring exenteration, and early colon or rectal (TEM) polyp cancers.

The structure of the MDT was adapted. Pre COVID, patients were booked to an individual surgeon clinic by the colorectal nurse specialists following the weekly MDT. During COVID, twice-weekly MDTs arranged via Microsoft teams allowed patients to be booked the next day into one of two multi-surgeon clinics offering a 'one stop shop' to meet a surgeon, discuss risks and sign consent, perhaps a second opinion, undergo preoperative anaesthetic assessment, obtain bowel prep and see a stomatherapist.

\section{Cancer priorities panel}

A cancer priorities panel meeting three times weekly was setup to match all cancer demand with theatre capacity. Initially, capacity constraints were high due to some theatre staff being required to self-isolate. In practice, this meant list availability being determined day to day, with the obvious need to be adaptive and dynamic in surgical planning.

Each surgical specialty was asked to keep a live list of surgical cancer patients in order of priority, as determined by an agreed classification that considered both likelihoods of cure (P1-4) and susceptibility to COVID (V1-3) [9].

There were ethics committee and ITU representation on the panel. The panel kept a real-time rate of elective COVID infections, with a numerator fed in weekly from all inpatient COVID testing, over a rolling denominator of cases done from the different surgical specialties. This allowed real-time discussion of the risk of contracting COVID after elective surgery to be discussed in the clinic, on top of other risk-benefit discussions.

\section{Colorectal planning and surgery}

This process was by necessity dynamic. A small team of colorectal surgeons met each morning to review theatre allocation, the list of cancer patients and surgical staff availability. Prediction modelling using Public Health England data suggested the possibility of ITU capacity being reached by mid-April, with the risk that theatres might be re-purposed to provide additional ITU beds [12]. We therefore agreed on some principles, largely to bring forward as much cancer surgery as possible before this potential re-purposing: dual consultant operating; open surgery; strong consideration for stomas to avoid anastomotic complications. This group developed strong singleconsultant portals into other disciplines including ITU, preop assessment and anaesthetics, to enable rapid communication and flexibility.

We initially paused laparoscopic surgery, following recommendations from the RCS and ACPGBI [8], as the situation became clearer, we resumed laparoscopy with additional precautions to avoid generating an aerosol, although evolving evidence suggests this is an unlikely source of infection in patients undergoing elective cancer surgery. We benefitted from guidance on the protection of anaesthetic staff during endotracheal intubation [13] but faced with a lack of definitive national guidance on PPE for theatre staff [14], we offered all theatre scrub staff level 2 PPE and provided extensive simulation training. Since the 
introduction of preop routine COVID swabbing, we reverted to level 1 PPE for theatre staff after intubation. This has allowed us to rationalise the use of PPE to ensure adequate supply for high-risk areas such as emergency theatres and ITU. The Trust currently has a small surplus, but the situation will be continually monitored, should cases rise sharply in the coming weeks.

We continued to follow our enhanced recovery protocols and discharged patients as early as safely possible, with follow-up by telephone as a 'virtual ward round'.

\section{COVID screening}

Our specific COVID protocol included all patients shielding for 14 days preop, drive-through phlebotomy and COVID swabbing, and deferral of patients exposed to symptomatic individuals within 14 days. Those undergoing major surgery were assessed using $\mathrm{CT}$ chest on the morning of surgery [15] together with symptom screening. Latterly, preoperative COVID swabbing $48 \mathrm{~h}$ before admission superseded CT chest for all patients. These simple practices need a robust infrastructure and rigorous adherence to practice, which sometimes meant surgery deferral on the day.

\section{Data collection}

In a prospective cohort study, we analysed all colorectal cases operated on at our Trust during the 11 weeks following the national lockdown on 23rd March. We compared the workload to the same 11-week period in 2019. The information was obtained from prospectively kept databases. Demographic information, disease aetiology, operation type and laparoscopic completion rates were collected and compared between the two cohorts. The primary outcomes of interest were length of hospital stay, complications and 30-day mortality.

A qualitative assessment of cancer treatment in the two cohorts was made. TMN staging, use of neoadjuvant chemoradiotherapy, R1 resection rates and lymph node yields among the cancer resections were analysed and compared between the cohorts. MDT discussions were reviewed to determine the extent of changes to treatment plans made in response to the COVID-19 pandemic.

We also analysed the precautions put in place to avoid perioperative COVID-19 infection, including preoperative screening (CT chest and swab testing), postponements and cancellations.

Chi 2 tests were used for categorical data and Man Whitney $\mathrm{U}$ tests for continuous data.

We also interrogated Trust and NBOCAP data to determine the monthly lower GI 2-week wait referral patterns and the total number of colorectal cancer resections and TEMS procedures performed between January 2019 and November 2020, to give a broader picture of the current status of our cancer service.

\section{Results}

\section{Preoperative preparation and theatre performance}

Figure 1 shows the change in the proportion of patients screened using CT chest and viral swabbing over the 11-week period. Initially all major resections underwent CT chest on the morning of surgery, however, as viral swabbing became available, all patients were swabbed 24-48 h preop. In week 9, the policy on CT chests changed and they were no longer performed routinely.

There were four on the day cancellations during the COVID period; one due to a delay in swab result, two due to lack of list capacity (reduced due to the increased turnaround time required for the donning and doffing of level II PPE) and one due to minor respiratory symptoms (the patient subsequently tested negative for COVID-19).

We saw a drop in our theatre efficiency across general surgery during the COVID period-averaging 56.8\%, compared to $76.2 \%$ during the non-COVID period. However, this has steadily improved over time to near-normal levels. (Fig. 2).

\section{Case load and demographics}

There were 85 operations performed in the 11 weeks following UK lockdown on $23^{\text {rd }}$ March 2020 (COVID cohort). A total of 179 procedures were performed during the same period in 2019 (non-COVID cohort). The number

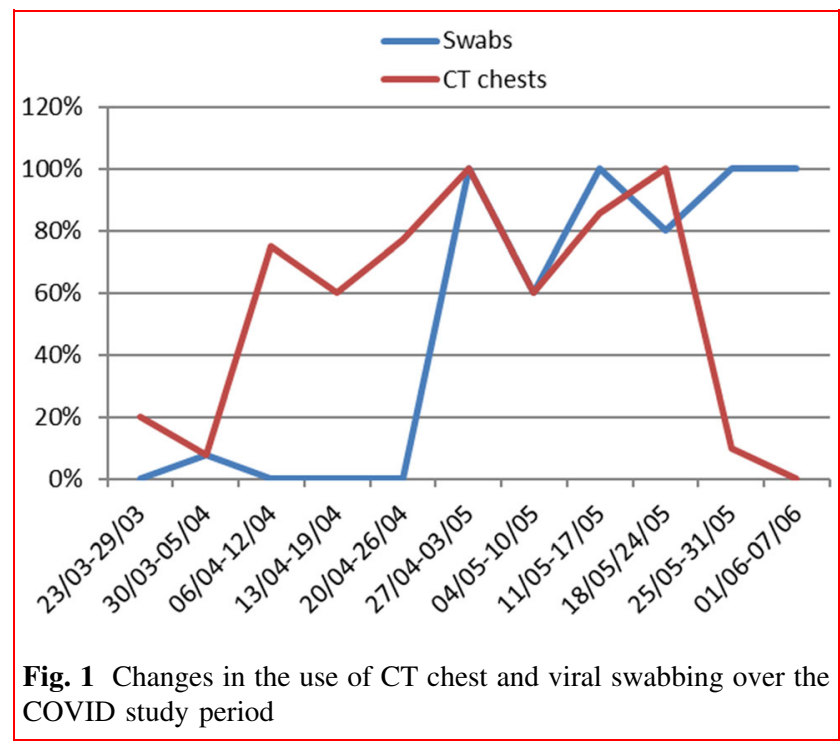




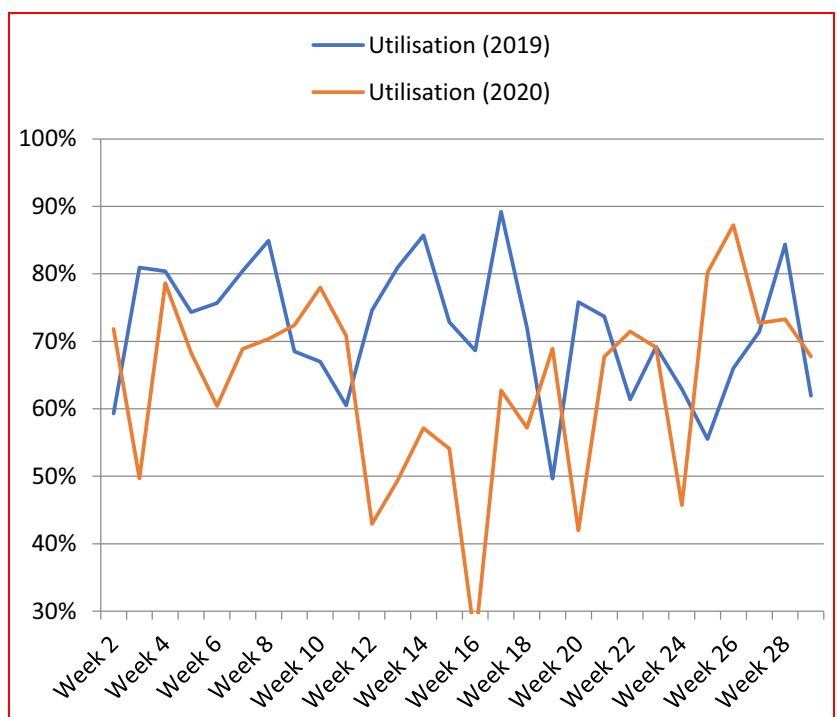

Fig. 2 Comparison of colorectal theatre utilisation (2019-2020)

of major colonic resections, however, was higher in the COVID cohort (53 vs 48 ). There was no significant difference in the proportion of right- vs left-sided resections $(p=0.88)$ (Table1). The additional cases in non-COVID cohort were for benign pathology and $83(46 \%)$ were day cases; in line with most other hospitals, all but the most urgent benign cases were postponed following UK lockdown [9].

Two-thirds of the COVID cohort were cancer cases, significantly more than in the Non-COVID cohort, where cancer only made up $26 \%$ of the workload $(p<0.00001)$. There were also more major cancer resections in the COVID cohort; 47 vs. 33 in the non-COVID cohort. Interestingly, the patients in the COVID cohort were significantly older, with an average of 63 years compared to 57 in the non-COVID cohort $(p=0.002)$. Moreover, a higher proportion of the COVID cohort patients were ASA

Table 1 Overview of caseload

\begin{tabular}{lrrr}
\hline & COVID & $\begin{array}{l}\text { Non- } \\
\text { COVID }\end{array}$ & $p$ value \\
\hline Total number of cases & 85 & 179 & \\
Number of colonic major resections & 53 & 48 & \\
Site & & & \\
Right sided & 22 & 23 & 0.88 \\
Left sided & 25 & 20 & \\
Subtotal & 2 & 1 & \\
Small bowel & 4 & 4 & \\
TEMS/TAMIS & 8 & 9 & \\
Coloproctology day case & 15 & 83 & \\
Other & 9 & 39 & \\
\hline
\end{tabular}

Table 2 Patient demographics

\begin{tabular}{lrrrrl}
\hline & \multicolumn{2}{l}{ COVID } & & Non-COVID & $p$ value \\
\hline $\begin{array}{l}\text { Mean age } \\
\text { (range) }\end{array}$ & $63(20-87)$ & & $57(19-89)$ & $\mathbf{0 . 0 0 2}$ \\
ASA 3+ & 30 & $35 \%$ & 36 & $20 \%$ & $\mathbf{0 . 0 0 7}$ \\
Cancer & 56 & $66 \%$ & 47 & $26 \%$ & $<\mathbf{0 . 0 0 0 0 1}$ \\
IBD & 11 & $13 \%$ & 37 & $21 \%$ & \\
Other benign & 18 & $21 \%$ & 95 & $53 \%$ & \\
\hline
\end{tabular}

Bold values are statistically significant for $p$ value

Table 3 Outcomes

\begin{tabular}{lrrrrc}
\hline & COVID & Non-COVID & $p$ value \\
\hline Median length of stay (days) & \multicolumn{1}{c}{4} & & 5 & & 0.09 \\
Readmissions & 5 & $6 \%$ & 14 & $8 \%$ & 0.56 \\
Clavien Dindo I \& II & 24 & $28 \%$ & 33 & $18 \%$ & 0.16 \\
Clavien Dindo III \& IV & 6 & $7 \%$ & 11 & $6 \%$ & \\
\hline
\end{tabular}

grade $3 ; 35$ versus $20 \%(p=0.007)$. (Table 2$)$ However, there was no significant difference in age or ASA grade among the cancer resections.

After excluding day cases, the median length of stay was 4 days in the COVID cohort and 5 days in the non-COVID cohort $(p=0.09)$. There was no significant difference in complications or readmissions between the two cohorts. There were no 30-day mortalities in either cohort. (Table 3).

\section{Cancer resections}

There were more cancer resections in the COVID cohort than the non-COVID cohort (47 vs 33 ), likely due to the cases we took from more severely affected neighbouring Trusts. A lower percentage of the COVID cohort cases had neoadjuvant chemoradiotherapy (19 vs 30\%), but this did not reach statistical significance.

Looking at the colorectal cancers, there was no difference in pathological TMN staging between the two cohorts. There were $2 \mathrm{R} 1$ resections in the COVID cohort (1 pelvic exenteration with deposits at the CRM but the primary fully excised and 1 with a metastatic deposit at the high tie after maximal excision) and 1 in the non-COVID cohort (tumour $0.9 \mathrm{~mm}$ from CRM in an APR). The mean lymph node yield was 23 (range 7-52) in the COVID cohort and 26 (range 8-52) in the non-COVID cohort $(p=0.27) .2$ (4\%) pathological specimens in the COVID cohort produced fewer than 12 nodes and $4(12 \%)$ in the non-COVID cohort $(p=0.188)$.

Overall, significantly more cases were performed open in COVID cohort ( 48 vs $26 \%, p=0.03$ ), due to our initial 
Table 4 Major resections for cancer

\begin{tabular}{|c|c|c|c|c|c|}
\hline \multirow[b]{2}{*}{ Total } & \multicolumn{2}{|c|}{ COVID } & \multicolumn{2}{|c|}{$\begin{array}{l}\text { Non- } \\
\text { COVID }\end{array}$} & \multirow[t]{2}{*}{$p$ value } \\
\hline & 47 & & 33 & & \\
\hline \multicolumn{6}{|l|}{ TMN stage } \\
\hline $\mathrm{T} 1$ & 5 & $10 \%$ & 5 & $15 \%$ & 0.24 \\
\hline $\mathrm{T} 2$ & 9 & $19 \%$ & 10 & $29 \%$ & \\
\hline $\mathrm{T} 3$ & 22 & $46 \%$ & 15 & $44 \%$ & \\
\hline $\mathrm{T} 4$ & 11 & $23 \%$ & 3 & $9 \%$ & \\
\hline No & 32 & $67 \%$ & 23 & $70 \%$ & 0.92 \\
\hline $\mathrm{N} 1+$ & 15 & $31 \%$ & 10 & $30 \%$ & \\
\hline R1 Resections & 2 & $4 \%$ & 1 & $3 \%$ & 0.77 \\
\hline $\begin{array}{l}\text { Mean Lymph node yield } \\
\text { (range) }\end{array}$ & 23 & $(7-52)$ & 26 & $(8-52)$ & 0.27 \\
\hline No. cases LN. yield $<12$ & 2 & $4 \%$ & 4 & $12 \%$ & 0.188 \\
\hline Open & 23 & $48 \%$ & 3 & $9 \%$ & $\mathbf{0 . 0 3}$ \\
\hline Laparoscopic & 24 & $50 \%$ & 25 & $74 \%$ & \\
\hline Conversions & 0 & $0 \%$ & 5 & $15 \%$ & \\
\hline Defunctioned & 13 & $28 \%$ & 11 & $33 \%$ & 0.54 \\
\hline Neoadjuvant CRT & 9 & $19 \%$ & 10 & $30 \%$ & 0.22 \\
\hline
\end{tabular}

Bold value are statistically significant for $p$ value

policy of avoiding laparoscopic surgery. However, there were no conversions in the COVID cohort and $5(15 \%)$ in the non-COVID cohort. The percentage of cases performed open was $80 \%$ during the first 2 weeks of the COVID data collection period, but after this, with appropriate precautions in place, laparoscopic surgery was reintroduced and the proportion of patients having laparoscopic surgery was more in line with, though still lower than the non-COVID cohort.

About $27 \%$ and $33 \%$ patients undergoing a major resection were given a defunctioning stoma in the COVID and non-COVID cohorts, respectively $(p=0.54)$. (Table 4).

Looking at an overview of colorectal cancer resections over a longer period, there was a decrease in the number of cancer resections performed in May, June and July 2020, but numbers have now increased and exceeded the 2019 numbers (Fig. 3). The fall in cancer resections likely reflects the drop in lower GI 2-week wait referrals seen in April and May 2020 (Fig. 4).

\section{MDT decision-making}

On analysis of the MDT decisions for cancer patients, ten patients $(21 \%)$ operated on during the COVID period had their treatment plan explicitly altered to take COVID into consideration. Eight patients who would ordinarily be considered for adjuvant chemotherapy were recommended for surgical follow-up only, as it was felt that the risks of
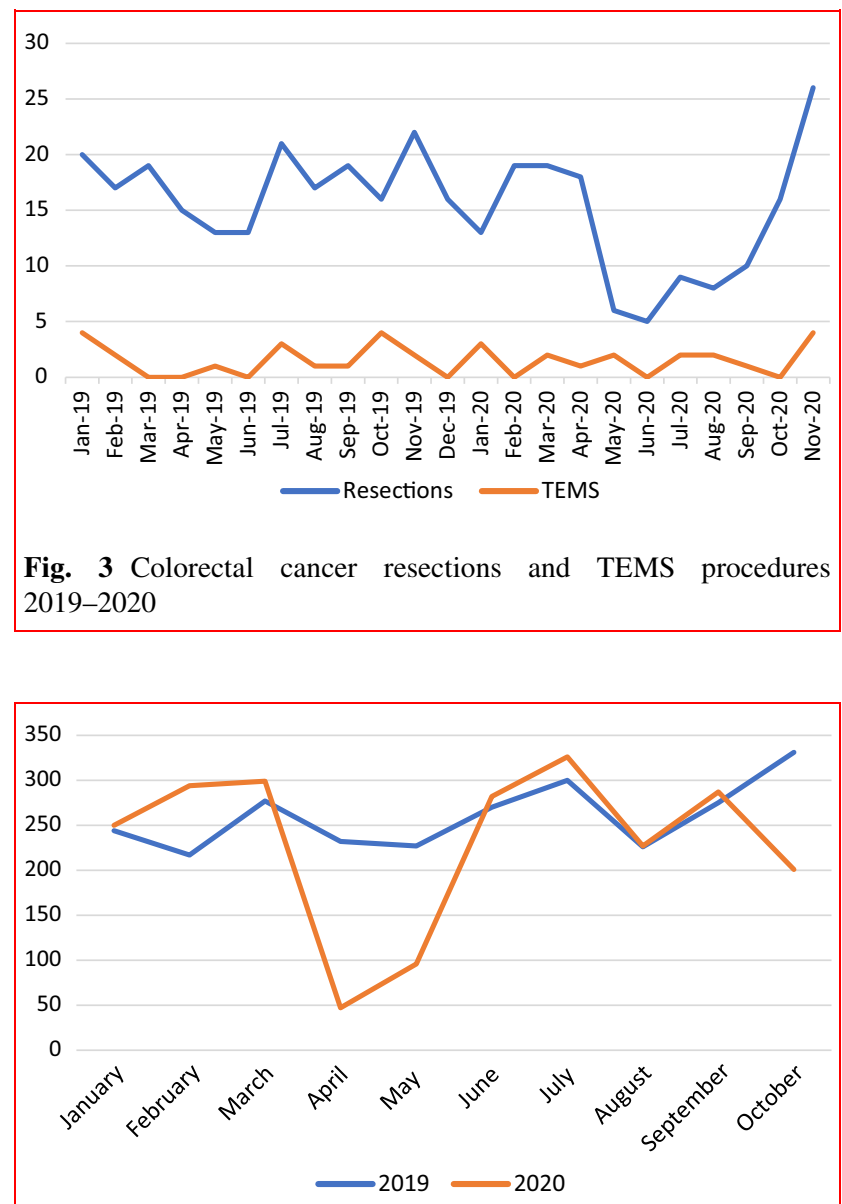

Fig. 4 Lower GI 2-week wait referrals to the Trust 2019-2020

chemotherapy would outweigh the benefits during the COVID-19 pandemic. Two patients had modified surgery; One patient had a less extensive resection (right hemicolectomy instead of a total colectomy in the context of a likely polyposis syndrome). One patient did not have an anastomosis (transverse colectomy). Both patients had significant co-morbidities.

In contrast to many centres, we did not resort to use of short-course radiotherapy as a 'holding treatment' for all cases of rectal cancer, however, in a proportion of those with an indication for neoadjuvant radiotherapy we opted for 25 Gy over 5 days (short course radiotherapy-SCRT) rather than chemoradiotherapy. Eight patients with primary rectal cancer discussed during the COVID period had short course chemoradiotherapy, where long course CRT would usually have been recommended 6 patients, the risk-benefit analysis was deemed to be in favour of standard longcourse CRT. Of the eight patients referred for SCRT, five completed the course and have since had resections, two had long course CRT following review in the oncology clinic and have not yet to had surgery and one has since 
developed liver and lung metastasis and been commenced on palliative chemotherapy.

Since June 2020, there has been a gradual shift back to standard long course chemoradiotherapy for all suitable patients. In the event of a second surge, each patient will be considered individually and those most at risk may once again be considered for short course CRT. However, with growing confidence in our 'clean' site setup, the threshold would now be higher.

There were very few patients deferred during the COVID study period. One patient requiring a pelvic exenteration was deferred for 3 months but has since had surgery. One frail patient requiring a TEMS for a tubulovillous adenoma was deferred and will return for a further clinic review next month.

\section{Discussion}

This study confirms that we continued to provide highquality care to cancer patients, even during the height of the UK pandemic. We were able to offer cancer surgery to all suitable candidates in our catchment area and take on cases from neighbouring Trusts who were unable to offer a Covid-19 free site. The short-term follow-up data show no increase in complications and readmissions. Importantly, the quality of the resections has also not been compromised, with no difference demonstrated in R1 resection rates and lymph node yields between the two cohorts.

In line with other UK centres, the re-organisation of services to keep elective cases at a 'clean' site and the precautionary steps laid out in the introduction has been crucial in avoiding both preoperative COVID-19 infections in elective patients and poorer outcomes, which may have been the result of delaying treatment. Clinicians need to have sensible discussions about risk with patients based on the latest available evidence [7, 16]. We can minimise, but not eliminate the risk of perioperative COVID-19 infection and this should be included in the standard consent process, until such time when a national vaccination programme has been fully implemented.

Ongoing close follow-up of this cohort is essential to assess the impact of acute decision-making, especially in terms of the reduction in the use of neoadjuvant and adjuvant chemoradiotherapy. This will be important during the ensuing months, as the risk of COVID-19 infection is likely to have an impact on decision-making for some time to come, and there is understandable concern about the risks of multiple hospital visits and immunosuppression associated with chemotherapy regimens [17].

Some practices from the process of adaption deserve to be taken forward beyond the pandemic, As a unit, we have already seen the advantages of streamlining the MDT process, both in terms of potential efficiency savings and in reducing hospital exposure for our most vulnerable patients. We have also seen how technology can be harnessed to make clinical decisions making across sites and speciality more inclusive and immediate, by removing the need for attendance in person. Some clinic appointments will likely continue to be conducted via Telemed, particularly routine follow-up and suitable new referrals who need triage prior to investigation. Feedback from patients on our Telemed service, especially those falling into the vulnerable category, has been excellent.

As we look to the immediate recovery phase, it is imperative that we resume effective diagnostic endoscopy and radiology services, as well as encourage patients, who are undoubtedly concerned about the risks of contact with medical services, to present with suspicious symptoms. A recent work from the Institute of Cancer Research predicts a devastating increase in mortality associated with a hypothetical 6-month delay in cancer treatment [18]. Our 2-week wait referral numbers fell significantly during the first surge. Although they have now returned to the numbers seen in 2019, there is still a 'missing' cohort who may not yet have come to the attention of secondary care.

Teamwork, agile decision-making and implementation of relevant national guidance with local expertise will help provide assurance that we can safely manage our diagnostic and treatment pathways and thus prevent a peak of avoidable deaths in the coming months.

From our experience, we are now much better prepared for the anticipated second surge of COVID-19 and will be able to immediately reinstate measures to keep our staff and patients safe and mitigate the impact on provision of elective services.

Acknowledgements We would like to acknowledge all those involved in the delivery of cancer surgery, as well as our patients who placed extraordinary faith in healthcare providers at this difficult time.

Funding None.

Compliance with ethical standards

Conflict of interest Authors declare that they have no conflict of interest.

\section{References}

1. Coronavirus Declared Pandemic by World Health Organization: WSJ [Internet]. Available from: https://www.wsj.com/articles/us-coronavirus-cases-top-1-000-11583917794

2. NHS England and NHS Improvement (2020). Letter to chief executives of all NHS trusts and foundation trusts, CCG accountable officers, GP practices and primary care networks, and providers of community health services 
3. Cancer referrals down by 80 percent in some areas as coronavirus fears keep patients from hospitals [Internet]. Available from: https://www.telegraph.co.uk/news/2020/04/15/cancer-referrals80-per-cent-areas-coronavirus-fears-keep-patients/

4. Mahase E. Covid-19: Urgent cancer referrals fall by $60 \%$, showing "brutal" impact of pandemic. BMJ 369: m2386. Available from: www.gov.uk/government/statistics/waiting

5. Nepogodiev D, Bhangu A (2020) Elective surgery cancellations due to the COVID-19 pandemic: global predictive modelling to inform surgical recovery plans. Br J Surg. https://doi.org/10. 1002/bjs. 11746

6. Lei S, Jiang F, Su W, Chen C, Chen J, Mei W et al (2020) Clinical characteristics and outcomes of patients undergoing surgeries during the incubation period of COVID-19 infection. EClinicalMedicine 1:21

7. Archer JE, Odeh A, Ereidge S, Salem HK, Jones GP, Gardner A, et al. (2020) Mortality and pulmonary complications in patients undergoing surgery with perioperative SARS-CoV-2 infection: an international cohort study. Lancet. Available from: http://www. thelancet.com/article/S014067362031182X/fulltext

8. The Association of Coloproctology of Great Britain and Ireland Considerations for Multidisciplinary Management of Patients with Colorectal Cancer during the COVID-19 Pandemic (2020)

9. Clinical guide to surgical prioritisation during the coronavirus pandemic: Royal College of Surgeons. Available from: https:// www.rcseng.ac.uk/coronavirus/surgical-prioritisation-guidance/

10. Abdelrahman T, Beamish A, Brown C, Egan R, Evans T, Ryan Harper E et al (2020) Surgery during the COVID-19 pandemic: operating room suggestions from an international Delphi process. Br J Surg. https://doi.org/10.1002/bjs.11747

11. Ives J, Huxtable R (2020) Surgical ethics during a pandemic: moving into the unknown? Br J Surg. https://doi.org/10.1002/bjs. 11638

12. Deasy J, Rocheteau E, Kohler K, Stubbs DJ, Barbiero P, Cresham $\mathrm{M}$, et al. Forecasting Ultra-early Intensive Care Strain from
COVID-19 in England. https://doi.org/10.1101/2020.03.19. 20039057

13. Cook TM, El-Boghdadly K, McGuire B, McNarry AF, Patel A, Higgs A (2020) Consensus guidelines for managing the airway in patients with COVID-19: Guidelines from the Difficult Airway Society, the Association of Anaesthetists the Intensive Care Society, the Faculty of Intensive Care Medicine and the Royal College of Anaesthetists. Anaesthesia 75(6):785-99

14. Puntis J (2020) Covid-19: questions remain over UK government's PPE guidance [Internet]. The BMJ. BMJ Publishing Group. 369. Available from: https://www.bmj.com/content/369/ bmj.m1964

15. Intercollegiate Guidance for Pre-Operative Chest CT imaging for elective cancer surgery during the COVID-19 Pandemic. Available from: https://www.rcsed.ac.uk/news-public-affairs/news/2020/april/ intercollegiate-guidance-for-pre-operative-chest-ct-imaging-forelective-cancer-surgery-during-the-covid-19-pandemic

16. Kasivisvanathan V, Lindsay J, Rakhshani-moghadam S, Elhamshary A, Kapriniotis K, Kazantzis G, et al. (2020) Evaluation of 30-day mortality for 500 patients undergoing nonemergency surgery in a COVID-19 cold site within a multicentre regional surgical network during the COVID-19 pandemic. Medrxiv. Available from: http://medrxiv.org/cgi/content/short/ 2020.06.10.20115543

17. Williams M, Mi E, le Calvez K, Chen J, Pakzad-Shahabi L, Dadhania S, et al. Estimating the Risk of Death from COVID-19 in Adult Cancer Patients. Clinical Oncology 2020; Available from: https://doi.org/10.1016/j.clon.2020.10.021

18. Sud A, Jones M, Broggio J, Loveday C, Torr B, Garrett A et al (2020) Collateral damage: the impact on cancer outcomes of the COVID-19 pandemic. Medrxiv. https://doi.org/10.1101/2020.04. 21.20073833

Publisher's Note Springer Nature remains neutral with regard to jurisdictional claims in published maps and institutional affiliations. 